\title{
THREE-GROUPS WITH CYCLIC CENTRE AND CENTRAL QUOTIENT OF MAXIMAL CLASS
}

\author{
S. B. CONLON \\ (Received 13 September; revised 30 November 1976) \\ Communicated by M. F. Newman
}

\begin{abstract}
All three-groups with cyclic centre and such that the quotient by the centre has maximal class are listed and a presentation with generators and relations for each is given.
\end{abstract}

Let $G$ be a $p$-group with cyclic centre $Z$ and such that $G / Z$ has maximal class. It is known (though not in the literature) and not difficult to verify that the analysis of $p$-groups of maximal class as set out in Blackburn (1958) or Chapter III, Section 14 of Huppert (1967) carries through for such a group $G$; one works with the upper central series instead of the lower central series in the maximal class case. Let

$$
\text { (1) }=G_{a+1}<Z=G_{a}<G_{a-1}<\ldots<G_{2}<G
$$

be the upper central series of $G$, where $a$ is the class of $G$. When $a=2$ or $3, G$ has an abelian maximal subgroup and is readily described (Conlon (1976)). For $a \geqslant 4$, we have that

$$
C_{G}\left(G_{2} / G_{4}\right)=\ldots=C_{C}\left(G_{a-2} / G_{a}\right)
$$

and this is a maximal subgroup $G_{1}$ of $G$. The subgroup $C_{G}\left(G_{a-1}\right)$ is also maximal and $G$ is called exceptional if $G_{1} \neq C_{G}\left(G_{a-1}\right)$. The quotient $G / Z$ is always a nonexceptional maximal class group. $G$ is not exceptional if $a$ is even. Further if $s \in G-G_{1}-C_{G}\left(G_{a-1}\right)$, then $s^{p} \in Z$. If $s_{1} \in G_{1}-G_{2}$, and if $s_{i+1}=\left[s_{i}, s\right](i=1, \ldots, a-1)$, then $G_{i}=\left\langle G_{i+1}, s_{i}\right\rangle$. All maximal subgroups of $G$ which contain $Z$ (and so $G_{2}$ ) and which are not equal to $G_{1}$ or $C_{G}\left(G_{a-1}\right)$ are also nonexceptional groups with centre $Z$. If $p=2$, then $G_{1}$ is abelian and $G$ is described in Conlon (1976).

When $p=3$ and $|Z|=3$ ( $G$ has maximal class $a$ ), then $G_{1}^{\prime}=\left[G_{2}, G_{1}\right] \leqslant Z=G_{a}$, $G$ is nonexceptional and such groups are described by Blackburn (1958). But if $|Z| \geqslant 3^{2}$, then $G$ can be exceptional. However if $G=G / Z$, then $G$ is of maximal class $a-1$ and so $\left[G_{2}, G_{1}\right] \leqslant G_{a-1}$, whence $\left[G_{2}, G_{1}\right] \leqslant G_{a-1}$. Thus $\left[s_{2}, s_{1}\right]=s_{a-1}^{f} z$ for $0 \leqslant f<3$ and $z \in Z$. Thus $G$ is certainly a quotient of a group with a presentation:

$$
\begin{gathered}
G(a)=\left\langle s, s_{1}, \ldots, s_{a}, Z\right| Z \text { is a central cyclic 3-group, } \\
s_{i+1}=\left[s_{i}, s\right](i=1, \ldots, a-1), s^{3}=z_{1},\left(s s_{1}^{-1}\right)^{3}=z_{2}, \\
\left.\left[s_{2}, s_{1}\right]=s_{a-1}^{t} z \text {, where } s_{a}, z_{1}, z_{2} \text { and } z \in Z\right\rangle .
\end{gathered}
$$


When $a=2$ or 3 , one can further suppose that $f=0$ and $z=1$. We have the following consequences (2),..,(9) of the relations in (1):

$$
\left[s_{3}, s_{1}\right]=s_{a}^{f} z^{-3} \text {. }
$$

Other than (2) and the relation $\left[s_{2}, s_{1}\right]=s_{a-1}^{f} z$, we have the following for $1 \leqslant i<j \leqslant a$ :

$$
\left[s_{j}, s_{i}\right]=z^{m(j-i) \cdot 3^{[(i+j-2) / 2]}},
$$

where $m(l+12)=m(l)$ for all $l \geqslant 0$ and for $0 \leqslant l<12$ we have:

$$
\begin{array}{rrrrrrrrrrrrr}
l & 0 & 1 & 2 & 3 & 4 & 5 & 6 & 7 & 8 & 9 & 10 & 11 \\
m(l) & 0 & 1 & -1 & 2 & -1 & 1 & 0 & -1 & 1 & -2 & 1 & -1
\end{array}
$$

In particular $m(2 l+1) \equiv(-1)^{l}(\bmod 3)$, for $l>0$.

$$
z^{3^{([a-1) / 2]}}=1 \text {. }
$$

(5) If $\left[s_{a-1}, s_{1}\right] \neq 1$, then $a$ is odd and $z$ has order exactly $3^{(a-1) / 2}$. Also in this case we have:

$$
\left[s_{a-1}, s_{1}\right]=z^{(-3)^{(a-3) / 2}} .
$$

(6) If $f \neq 0$, then $\left[s_{a-1}, s_{1}\right]=s_{a}^{-1}$.

$$
\begin{gathered}
{\left[s_{a-1}, s_{a-2}\right]=\ldots=\left[s_{a-1}, s_{2}\right]=1 .} \\
s_{1}^{3}=\left(s_{3}^{-1}\right)^{s^{-1}} s_{a-1}^{-f} z^{2} z_{1} z_{2}^{-1}=s_{4} s_{6} \ldots s_{5}^{-1} s_{3}^{-1} s_{a-1}^{-f} z^{2} z_{1} z_{2}^{-1} .
\end{gathered}
$$

For $i>1$,

For $a \geqslant 3$,

$$
s_{i}^{3}=\left(s_{i+2}^{-1}\right)^{8^{-1}} z^{2.3^{t-1}}=s_{i+3} s_{i+5} \ldots s_{i+4}^{-1} s_{i+2}^{-1} z^{2.33^{i-1}} .
$$

$$
\begin{gathered}
s_{a-1}^{3}=1 . \\
s_{a}^{3}=1 .
\end{gathered}
$$

By passing to the quotient $G(a) / Z$ and using induction on $a$, it is readily seen that $G(a)$ is a 3-group and that $|G(a)| \leqslant|Z| \cdot 3^{a}$.

Relations (4), (5), (6) and (9) place restrictions on the choice of $z$ and $s_{a}$ in $Z$. Once these are satisfied, then $|G(a)|=|Z| .3^{a}$ without any collapse and $G(a)$ gives the correct form of our sought group $G$ of class $a$. To see this one constructs by induction on $a$ the group $G(a+1)$ from a maximal subgroup $G(a)$ by adding another element $s_{0}$ satisfying:

$$
\left[s_{0}, s\right]=s_{1}, \quad\left[s_{1}, s_{0}\right]=s_{a-1}^{f^{\prime}} z^{\prime},
$$

and $s_{0}$ acts identically on $Z$. $s_{0}$ then gives an automorphism of $G(a)$ provided

$$
z^{3[(a-2) / 2]}=1, f=0, \quad\left(z^{\prime}\right)^{3}=z \quad \text { and } z_{1}=z_{2} .
$$

To assign a value to $s_{0}^{3}$, we choose instead to insist that $\left(s s_{0}^{-1}\right)^{3} \in Z$ and $\left(s s_{0}^{-1}\right)^{3}$ acts identically on $G(a)$ provided

$$
\left(s_{a}\left[s_{1}, s_{a-2}\right]\right)^{f^{\prime}}=1
$$


The conditions (11) and (12), with $s_{i}$ replaced by $s_{i+1}$ hold in any case in the maximal subgroup $\left\langle s, s_{2}, \ldots, s_{a+1}, Z\right\rangle$ of a group $G(a+1)$ defined as in (1).

The fact that $Z$ is the centre of $G(a)$ and that $G(a) / Z$ has maximal class $a-1$ is proved by induction on $a$.

The isomorphism problem is resolved by looking at all possible choices of generators $s, s_{1}$ and $t$ and pushing $G=G(a)$ into a standard form; here $t$ is a generator of the centre $Z$. In separating the isomorphism classes, account may also be taken of the number $h$ of maximal subgroups $M$ of $G$ such that $G_{2} \leqslant M$ and $M \neq G_{1}$ or $C_{G}\left(G_{a-1}\right)$ and such that every element in $M-G_{2}$ has order 3.|Z|.

$a$ is the class of $G, 3^{b}=|Z|$ and $3^{c}=\left|G_{1}^{\prime}\right|$. We can suppose that $z=t^{g .3^{b-c}}$, where $g \equiv \pm 1(\bmod 3)$. In the nonexceptional cases we can always make $g=1$. The exceptional cases occur when $c=[a / 2]$ and $a$ is odd and greater than 4; here we can insist that $s s_{1} \in C_{G}\left(G_{a-1}\right)$ which implies that $\left[s_{a-1}, s_{1}\right]=s_{1}^{-1}$ and that $g \equiv(-1)^{(a-1) / 2}(\bmod 3) . G$ is presented as follows:

$$
\begin{gathered}
\text { Gabcdef }=\left\langle s, s_{1}, \ldots, s_{a}, t\right|\left[s_{i}, s\right]=s_{i+1}(i=1, \ldots, a-1), \\
{[t, s]=\left[t, s_{1}\right]=1, t^{b^{b-1}}=s_{a}, s^{3}=t^{d},} \\
\left(s s_{1}^{-1}\right)^{3}=t^{e},\left[s_{2}, s_{1}\right]=s_{a-1}^{t} t^{\left.g .3^{b-c}\right\rangle .}
\end{gathered}
$$

The table (14) gives the values of the parameters to obtain a full set of nonisomorphic groups. The values of $g$ and $h$ are also included. The values of $d, e, f$ and

\begin{tabular}{|c|c|c|c|c|c|c|c|c|}
\hline & $a$ & $b$ & $c$ & $d$ & $e$ & $f$ & $g$ & $h$ \\
\hline \multirow[t]{3}{*}{ Non-exceptional } & $\begin{aligned} & \geqslant 2 \\
& \geqslant 2 \\
\text { odd } & \geqslant 3\end{aligned}$ & $\begin{array}{l}\geqslant 1 \\
\geqslant 1 \\
\geqslant 1 \\
\geqslant 1\end{array}$ & $\begin{array}{l}\mathbf{0} \\
0 \\
0 \\
0\end{array}$ & $\begin{array}{l}0 \\
1 \\
0 \\
0\end{array}$ & $\begin{array}{l}0 \\
1 \\
1 \\
2\end{array}$ & $\begin{array}{l}0 \\
0 \\
0 \\
0\end{array}$ & $\begin{array}{l}1 \\
1 \\
1 \\
1\end{array}$ & $\begin{array}{l}0 \\
3 \\
2 \\
2\end{array}$ \\
\hline & $\begin{array}{l}\geqslant 4 \\
\geqslant 4 \\
\geqslant 4\end{array}$ & $\begin{array}{l}b=c \\
b=c \\
b=c\end{array}$ & $\begin{array}{l}0<c<[a / 2] \\
0<c<[a / 2] \\
0<c<[a / 2]\end{array}$ & $\begin{array}{l}0 \\
0 \\
1\end{array}$ & $\begin{array}{l}0 \\
2 \\
1\end{array}$ & $\begin{array}{l}0 \\
0 \\
0\end{array}$ & $\begin{array}{l}1 \\
1 \\
1\end{array}$ & $\begin{array}{l}1 \\
2 \\
3\end{array}$ \\
\hline & $\begin{aligned} & \geqslant 4 \\
& \geqslant 4 \\
& \geqslant 4 \\
& \geqslant 4 \\
\text { odd } & \geqslant 5\end{aligned}$ & $\begin{array}{l}b>c \\
b>c \\
b>c \\
b>c \\
b>c\end{array}$ & $\begin{array}{l}0<c<[a / 2] \\
0<c<[a / 2] \\
0<c<[a / 2] \\
0<c<[a / 2] \\
0<c<[a / 2]\end{array}$ & $\begin{array}{l}0 \\
1 \\
2 \\
0 \\
0\end{array}$ & $\begin{array}{l}0 \\
1 \\
2 \\
1 \\
2\end{array}$ & $\begin{array}{l}0 \\
0 \\
0 \\
0 \\
0\end{array}$ & $\begin{array}{l}1 \\
1 \\
1 \\
1 \\
1\end{array}$ & $\begin{array}{l}0 \\
3 \\
3 \\
2 \\
2\end{array}$ \\
\hline Exceptional & $\begin{array}{l}\text { odd } \geqslant 5 \\
\text { odd } \geqslant 5 \\
\text { odd } \geqslant 5 \\
\text { odd } \geqslant 5 \\
\text { odd } \geqslant 5 \\
\text { odd } \geqslant 5 \\
\text { odd } \geqslant 5 \\
\text { odd } \geqslant 5 \\
\text { odd } \geqslant 5 \\
\text { odd } \geqslant 5 \\
\text { odd } \geqslant 5 \\
\text { odd } \geqslant 5\end{array}$ & $\begin{array}{l}b \geqslant c \\
b \geqslant c \\
b \geqslant c \\
b \geqslant c \\
b \geqslant c \\
b \geqslant c \\
b \geqslant c \\
b \geqslant c \\
b \geqslant c \\
b \geqslant c \\
b \geqslant c \\
b \geqslant c\end{array}$ & $\begin{array}{l}c=[a / 2] \\
c=[a / 2] \\
c=[a / 2] \\
c=[a / 2] \\
c=[a / 2] \\
c=[a / 2] \\
c=[a / 2] \\
c=[a / 2] \\
c=[a / 2] \\
c=[a / 2] \\
c=[a / 2] \\
c=[a / 2]\end{array}$ & $\begin{array}{l}0 \\
0 \\
0 \\
0 \\
0 \\
0 \\
1 \\
1 \\
1 \\
1 \\
2 \\
2\end{array}$ & $\begin{array}{l}0 \\
0 \\
1 \\
2 \\
1 \\
2 \\
1 \\
1 \\
2 \\
2 \\
2 \\
2\end{array}$ & $\begin{array}{l}0 \\
1 \\
0 \\
0 \\
1 \\
1 \\
0 \\
1 \\
0 \\
1 \\
0 \\
1\end{array}$ & $\begin{array}{l}(-1)^{(a-1) / 2} \\
(-1)^{(a-1) / 2} \\
(-1)^{(a-1) / 2} \\
(-1)^{(a-1) / 2} \\
(-1)^{(a-1) / 2} \\
(-1)^{(a-1) / 2} \\
(-1)^{(a-1) / 2} \\
(-1)^{(a-1) / 2} \\
(-1)^{(a-1) / 2} \\
(-1)^{(a-1) / 2} \\
(-1)^{(a-1) / 2} \\
(-1)^{(a-1) / 2}\end{array}$ & $\begin{array}{l}0 \\
0 \\
1 \\
1 \\
1 \\
1 \\
2 \\
2 \\
2 \\
2 \\
2 \\
2\end{array}$ \\
\hline
\end{tabular}
$g$ are only significant modulo 3 .

TABLe (14) 


\section{References}

N. Blackburn (1958), "On a special class of p-groups", Acta Math. 100, 45-92.

S. B. Conlon (1976), "p-Groups with an abelian maximal subgroup and cyclic center", $J$. Austral. Math. Soc. 22, 221-233.

B. Huppert (1967), Endliche Gruppen I (Die Grundlehren der mathematischen Wissenschaften, 134. Springer-Verlag, Berlin, 1967).

\section{Department of Pure Mathematics}

University of Sydney

NSW 2006, Australia 